\title{
Molecular gas distribution and dynamics in the central region of galaxies
}

\author{
Sumio ISHIZUKI \\ Nobeyama Radio Observatory, National Astronomical Observatory, \\ also Department of Astronomy, Faculty of Science, University of Tokyo
}

\section{ABSTRACT}

To investigate the relation between a large-scale stellar bar and circumnuclear starburst, the CO $(J=1 \rightarrow 0)$ emission of circumnuclear regions of three starburst galaxies NGC2782, NGC3504, and M83 (NGC5236) have been mapped with the Nobeyama Millimeter Array. The high resolntion $C O(J=1 \rightarrow 0)$ images indicate that the molecular gas in the three starburst galaxies is located interior to the innermost ends of the paired straight dust lanes. The molecular gas at the small radii is associated with their circumnuclear starburst sites which are indicated by radio continuum emission.

\section{INTRODUCTION}

In many galaxies, the circumnuclear regions are sites of enhanced star formation or starburst. The gas is exhausted in a time scale of $\sim 10^{8}$ years due to starburst. Since the time scale is shorter than the age of galaxies, the problem of how the gas is transported to the nuclear starburst has to be addressed.

The gravitational potential field of a bar or of an oval shape is a strong candidate for a mechanism for fueling circumnuclear regions. A good example is IC342. The observations suggest that the gas is transported toward the nucleus by the effect of bar shocks and the starburst occurs in a small ring of molecular gas which has been formed at the radius where the gas infall stops (Ishizuki et al. 1990). The question of whether the structure of a bar and a circumnuclear ring is common in starburst galaxies still remained.

To investigate the mechanisms for fueling starburst nuclei of barred galaxies, the $\mathrm{CO}(J=1 \rightarrow 0)$ emission of the circumnuclear regions of three barred galaxies, NGC2782, NGC3504, and M83 (NGC5236) were observed with the Nobeyama Millimeter Array (NMA). Parameters for the observed galaxies are listed in Table 1. All the three galaxies have paired straight dust lanes which offset toward their leading sides of the large scale stellar bars. NGC2782 and NGC3504 are selected as starbursts by Balzano (1983). The evidence for starburst in M83 is suggested by Stacey et al. (1991), Bohlin et al. (1983), Trinchieri, Fabbiano, and Paulumbo (1985), and Ohashi et al. (1990). 
Table. 1 Parameters for observed starburst galaxies

\begin{tabular}{llll}
\hline & NGC2782 & NGC3504 & NGC5236 $^{-}$ \\
\hline Morphology $^{a}$ & SAB(rs)aP & SB(s)c & SAB(s)c \\
Distance & $34 \mathrm{Mpc}^{b}$ & $20 \mathrm{Mpc}^{b}$ & $3.7 \mathrm{Mpc}{ }^{\mathrm{e}}$ \\
P.A. & $81^{\circ \mathrm{c}}$ & $149^{\circ d}$ & $45^{\circ f}$ \\
inclination & $28^{\circ \mathrm{c}}$ & $22^{\circ d}$ & $13^{\circ f}$ \\
\hline
\end{tabular}

(a) de Vancouleurs et al. (1990). (b) Calculated from the Galactocentric velocities listed by de Vaucouleurs et al. (1990) and $H_{0}=75 \mathrm{~km}$ $\mathrm{s}^{-1} \mathrm{Mpc}^{-1}$. (c) van der Kruit (1977). (d) Grosb申l (1985). (e) de Vancouleurs (1979). (f) Rogstad, Lockhart, and Wright (1974).

\section{OBSERVATIONS AND RESULTS}

The observations wes made between January and May 1991 for NGC2782 and NGC3504, and between December 1990 and May 1991 for M83. The NMA (Ishiguro et al. 1984, Morita 1993) was used for the observations. It consists of five antennas. The FX spectrocorrelator covers $320 \mathrm{MHz}\left(830 \mathrm{~km} \mathrm{~s}^{-1}\right.$ at $\left.115 \mathrm{GHz}\right)$ simultaneously. The half power width of the primary beam is $65^{\prime \prime}$. The visibilities are calibrated with respect to $0923+392$ for NGC2782 and NGC3504 and to $1334-127$ for M83. The flux densities of the calibrators were measured with respect to Mars and Uranus.

NGC2782 A map of integrated CO $(J=1 \rightarrow 0)$ intensities is illustrated in Fig. 1. Two CO peaks are seen. The radial velocities, $V_{L S R}$, of the northeastern and southwestern peaks are $2387-2465$ and $2387-2465$ $\mathrm{km} \mathrm{s} \mathrm{s}^{-1}$, respectively. The peaks are located at radius $2^{\prime \prime} .5$ (or $400 \mathrm{pc}$ ). Their molecular masses derived from the CO fluxes are $0.9 \times 10^{9} M_{\odot}$ and $0.8 \times 10^{9} M_{\odot}$ for the northeastern and southwestern peaks, respectively $\left(M\left(\mathrm{H}_{2}\right)=1.1 \times 10^{4}(D / \mathrm{Mpc})^{2}\left(S_{\mathrm{CO}} / \mathrm{Jykms}^{-1}\right)\right.$ is assumed $)$. The total $\mathrm{CO}$ flux is $207 \mathrm{Jy} \mathrm{km} \mathrm{s}{ }^{-1}$, corresponding to the molecular mass of $2.6 \times$ $10^{9} M_{\odot}$. The southwestern CO peak has an extension toward the west to form a $\mathrm{CO}$ ridge. The $\mathrm{CO}$ ridge continues to the end of the straight dust lane at the northwest side of the large scale stellar bar (the upper right line in Fig. 1). Although the extension is in the same direction as the convolving beam, this extension is real since it is longer than the major axis of the synthesized beam (this is confirmed by a velocity channel map at $V_{L S R}=2660 \mathrm{~km} \mathrm{~s}^{-1}$ which does not appear in this paper).

Wilson and Willis (1980) have obtained a radio continuum map at $4885 \mathrm{MHz}$. It is composed of a nuclear peak, a pair of northeast and southwest peaks at radius $3^{\prime \prime}$ with position angle $60^{\circ}$, and other extended 


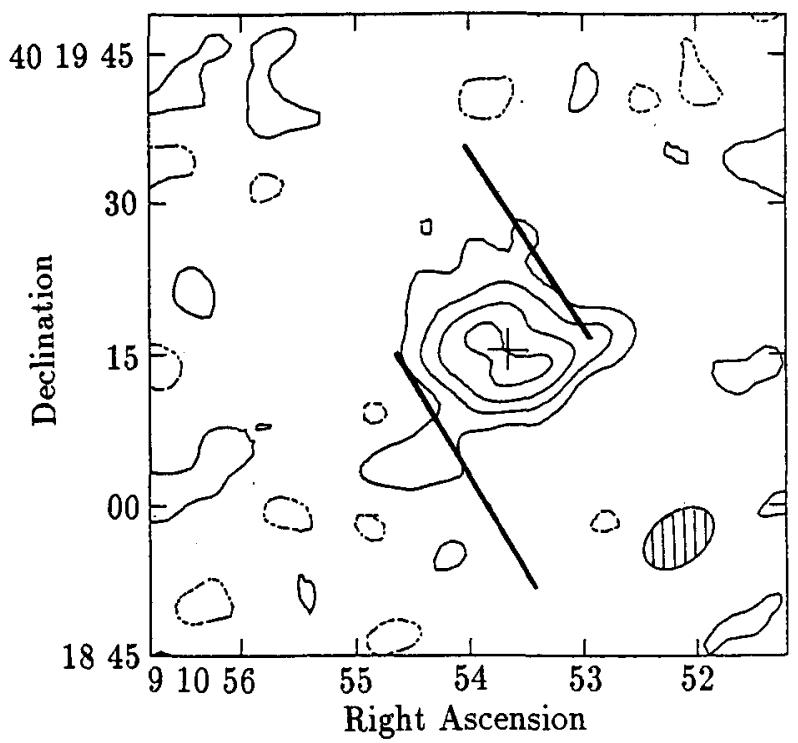

Figure $1 \mathrm{~A}$ map of integrated $\mathrm{CO}(J=1 \rightarrow 0)$ intensities in NGC 2782. The angular resolution obtained is $8^{\prime \prime} .0 \times 5 .{ }^{\prime \prime} 2$ with a position angle of $126^{\circ}$. The long thick lines represent dust lanes which lie along the leading sides of the large-scale stellar bar.

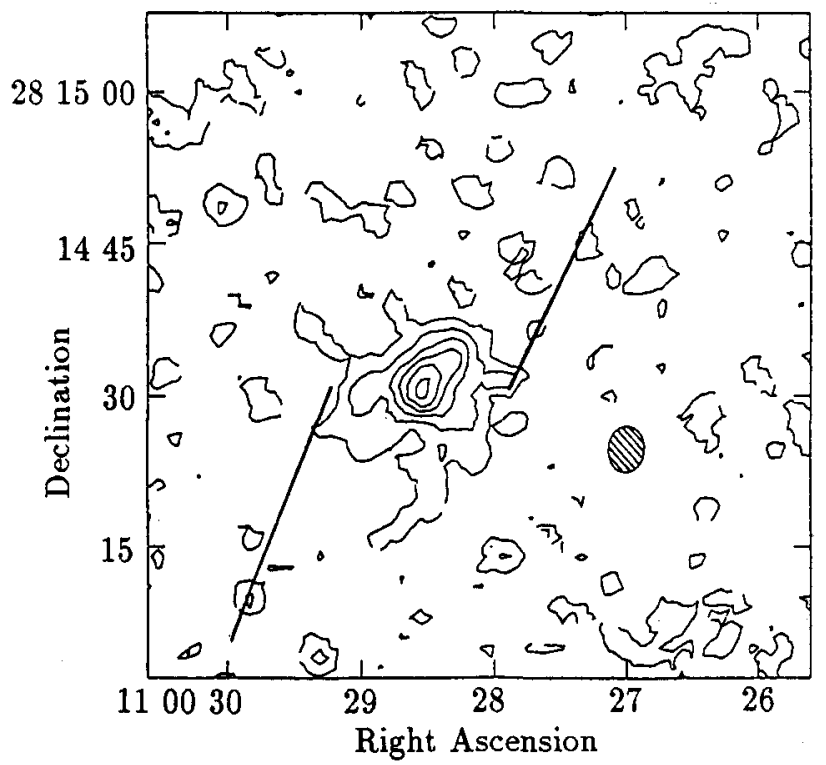

Figure 2 A map of integrated CO $(J=1 \rightarrow 0)$ intensities in NGC 3504. It is made with uniform weighting with a taper. The angular resolution obtained is $4^{\prime \prime} .7 \times 3 .^{\prime \prime} 7$ with a position angle of $176^{\circ}$. The long thick lines represent dust lanes on the large-scale stellar bar. 
components. The $\mathrm{CO}$ peaks are associated with the paired radio peaks at radius $3^{\prime \prime}$. This indicates that the nuclear starburst is associated with the CO peaks.

NGC3504 A map of integrated CO $(J=1 \rightarrow 0)$ intensities are illustrated in Fig. 2. The $\mathrm{CO}$ emission is located interior to the dust lanes on the large-scale stellar bar. The $\mathrm{CO}$ appears to elongate in the direction which is almost parallel to the large-scale stellar bar. The total CO flux is $415 \mathrm{Jy}$ $\mathrm{km} \mathrm{s}^{-1}$ in a map of natural weighting (data not provided), corresponding to the molecular mass of $1.8 \times 10^{\circ} M_{\odot}$. The CO flux from the compact components which appear in Fig. 2 is $280 \mathrm{Jy} \mathrm{km} \mathrm{s}^{-1}$. Superposition of the $\mathrm{CO}$ map on an $\mathrm{H} \alpha$ map given by Kenney et al. (1992) indicates that the starburst is associated with the CO component in Fig. 2.

M83 (NGC5236) Handa, Ishiznki, and Kawabe (1993, this issue) have investigated the $\mathrm{CO}$ in the circumnuclear $1 \mathrm{kpc}$ region of $\mathrm{M83}$ and their results are summarized as follows: (1) The $\mathrm{CO}$ distribution makes a barlike structure which connects the pair of major dust lanes on the largescale stellar bar. The orientation of the $\mathrm{CO}$ bar-like structure (hereafter the inner $\mathrm{CO}$ bar) is tilted relative to the orientation of the large-scale stellar bar. (2) The $\mathrm{CO}$ velocity field indicates that there are non-circular motions whose orientations are likely parallel to the inner CO bar. (3) The $6 \mathrm{~cm}$ radio continuum map by Cowan and Branch (1985) indicates an east-west elongation which suggests a pair of radio peaks at radii $6^{\prime \prime}$. The orientation of the peaks is perpendicular to the inner $\mathrm{CO}$ bar.

\section{DISCUSSION}

One characteristic of the three starburst galaxies is that the $\mathrm{CO}$ emission makes structures interior to the dust lanes. This situation is very different from the CO twin peak galaxies investigated by Kenney et al. (1992) although straight dust lanes at the leading sides of the large-scale stellar bars are a common feature of both types of galaxies. Not only the distribution of the $\mathrm{CO}$ emission, but the locations of star formation are different; the star formation, which is indicated by radio continuum emission, is associated with the CO concentrations which are interior to the dust lanes in the starburst galaxies, while it is associated with a ring which touches with the dust lanes in the $\mathrm{CO}$ twin peak galaxies.

The features of the inner CO bar of M83 are similar to those of the CO bar of IC342, where there are non-circular motions whose orientations are along the $\mathrm{CO}$ bar and a pair of radio peaks (which forms a ring) whose orientation is perpendicular to the CO bar (Ishizuli et al. 1990). As for IC342, it is suggested that the non-circular motion along the molecular bar feeds the circumnuclear starburst of M83. The CO distribution in the circumnuclear region of M83 is evidently different from the $\mathrm{CO}$ twin peak 
galaxies investigated by Kenney et al. (1992); M83 has the inner CO bar and the location where the two points of the inner $\mathrm{CO}$ bar of M83 are is the same as where the $\mathrm{CO}$ twin peaks would be if M83 had CO twin peaks.

In NGC2782, one of the paired CO peaks (the southwest peak), is elongated to form a $\mathrm{CO}$ ridge toward the west and continues to the end of the northwest dust lane. The intersection between the ridge and the dust lane is almost perpendicular. This relationship between the $\mathrm{CO}$ ridge and the dust lane in NGC2782 is similar to the relationship between the inner $\mathrm{CO}$ bar and the dust lanes in M83. The paired CO peaks are associated with the radio continu um peaks, which traces star forming regions, and the orientation of the $\mathrm{CO}$ peaks are tilted relative to the orientation of the $\mathrm{CO}$ ridge. This relationship between the $\mathrm{CO}$ ridge and the radio peaks in NGC2782 is similar to the relationship between the inner CO bar and the radio peaks in $\mathrm{M} 83$.

The molecular gas distributions of NGC2782 and M83 are interpreted in the following way. As investigated by many au thors (e.g. Contopoulos and Papayannopoulos 1980), non-self-intersecting closed orbits in a rotating frame of an oval potential field of a galaxy are divided into two kinds of families: the family of orbits whose orientations of the major axes are parallel to the major axis of the potential field ( $x_{1}$ family), and the family of orbits whose orientations of the major axes are perpendicular to the major axis of the potential field ( $x_{2}$ family). Their orientations change at Lindblad resonances and at a corotation resonance. The dynamics of gas clouds is well explained by non-self-intersecting closed orbits due to inelasticity. The gas in the dust lanes on a large-scale stellar bar is in the orbits of the $z_{1}$ family. The straight dust lanes ends at the outer inner Lindblad resonance (OILR) where the orientations of the orbits change. The gas in the inner $\mathrm{CO}$ bar of $\mathrm{M} 83$ and in the two $\mathrm{CO}$ peaks and the $\mathrm{CO}$ ridge of NGC2782 belongs to the orbits of the $x_{2}$ family inside an OILR. This interpretation is similar to that for the $\mathrm{CO}$ twin peaks described by Kenney et al. (1992), but the gas distributions in the $x_{2}$ orbits are different. The molecular gas is concentrated to pericenters in NGC2782 and located at the whole of the $x_{2}$ orbits in M83; the gas is concentrated within the OILRs. On the other hand, the gas is concentrated to the apocenters around the OILR in $\mathrm{CO}$ twin-peak galaxies.

One possible explanation of the structure of NGC2782 and M83 is the effect of the inner inner Lindblad resonance (IILR). According to Athanassoula (1992), the gradual change of the orientations of the orbits from the $x_{1}$ to the $x_{2}$ family around an OILR creates shock waves. When this idea is extended to the region around an IILR, it is expected that concentrations of gas are determined by the gradual change of the orientations of the orbits from the $x_{2}$ family (outside the IILR) to the $x_{1}$ 
family (inside the IIIR). Also, IILR may contribute to starburst; in both NGC2782 and M83, the orientation of radio continuum peaks are tilted relative to the $\mathrm{CO}$ ridge and the inner $\mathrm{CO}$ bar, respectively, implying $\boldsymbol{x}_{1}$ orbits: in NGC3504, the CO is concentrated far inside the dust lanes and elongated parallel to the large-scale stellar bar, and the $\mathrm{CO}$ concentration is at the site of starburst.

What makes the gas fall inside the dust lanes? One of the possibility is the presence of an IILR, as expected from the discussions in the last paragraph. Another is the self-gravity of the gas as indicated by Fukunaga and Tosa (1991) and Wada and Habe (1992). The gas mass fractions inside the inner ends of the straight dust lanes (corresponding to the OILR) are $9 \%$ and $16 \%$ in NGC2782 and NGC3504, respectively. These fractions are sufficient for self-gravity to affect the behavior of the gas (Fukunaga and Tosa 1991; Wada and Habe 1992).

\section{REFERENCES}

Athanassoula, E. 1992, MNRAS, 258, 345

Balzano V. A. 1983, ApJ, 268, 602

Bohlin, R. C. et al. 1983, ApJL, 274, L53

Contoponlos, G. and Papayannopoulos, Th. 1980, A\&A, 92, 33

Cowan, J. J. \& Branch, D. 1985, ApJ, 293, 400

de Vaucouleurs, G. 1979, AJ, 84, 1270

de Vaucouleurs, G. et al. 1991, Third Reference Catalogue of Bright Galaxies (New York: Springer-Verlag)

Fukunaga, M. and Tosa, M. 1991, PASJ43, 469

Grosb $\phi 1$, P. J. 1985, A\&AS, 60, 261

Handa, T., Ishizuki, S., and Kawabe, R. 1993, This issue

Ishiguro, M. et al. 1984, Proceeding of the International Symposium on Millimeter and Submillimeter Wave Radio Astronomy (ed. Gomez-Gonzales, J.) 75 (URSI, Granada)

Ishizuki, S. et al. 1990, Nature, 344, 224

Kenney, J. D. P. et al. 1992, ApJL, 395, L79

Morita, K. 1993, This issue

Ohashi, T. et al. 1990, ApJ, 365, 180

Ohashi, T., Makishima, K., Tsuru, T., Takano. S., Koyama, K., Stewart, G. C. 1990, ApJ, 365, 180

Rogstad, D. H.. Lockhart, I. A., and Wright, M. C. H. 1974, ApJ, 193, 309

Stacey, G. J. et al. 1991, ApJ, 373, 444

Trinchieri, G., Fabbiano, G., and Paulumbo, G. G. C. 1985, ApJ, 290, 96 van der Kruit, P. C. 1977, A\&A, 61, 171

Wada, K., and Habe, A. 1992, MNRAS, 258, 82

Wilson, A. S., and Willis, A. G. 1980, ApJ, 240, 429 


\section{QUESTIONS}

GENZEL: How does the position angle of $\mathrm{CO}$ emission in M83 as seen by the NMA compare with the highest single dish results?

ISHIZUKI: The elongation of CO emission as seen by the Nobeyama 45 meter telescope is nearly parallel to the large scale stellar bar (Handa et al. 1990, PASJ, 42,1). But the orientation of the CO elongation in the central 1 minute is different from the large scale stellar bar. The field of view of the NMA map is limited to the central 1 minute where the $\mathrm{CO}$ emission was not well resolved by Handa et al..

KENNEY: Have you computed the ratio of starburst luminosity to gas mass in NGC2782? If this galaxy is in an early phase of a starburst, this ratio might be lower than other starbursts, since not much of the gas mass would yet be consumed.

ISHIZUKI: The ratio for the center of NGC2782 corresponds to a gas consumption time $5 \times 10^{8}$ years. The ratio is lower than starburst galaxies NGC253, NGC3504, and NGC7714 by factors of $2.5,1.3$, and 3 , respectively, when the starburst luminosity is defined by the 20 centimeter continuum flux. The factors are 2.5 - 3 when the starburst luminosity is defined by the $\mathrm{H} \alpha$ flux. The ratio is higher than hotspot galaxies (NGC2903, NGC5383, NGC3351, and NGC6951) by factors of 3-4. The intermediate value of NGC2782 may suggest that NGC2782 is at the intermediate stage between starburst galaxies and hotspot galaxies. 